\begin{tabular}{ccc}
\hline & International Journal of Engineering \& Technology, $7(2.12)(2018) 276-280$ \\
SPC & International Journal of Engineering \& Technology \\
Website www.sciencepubco.com/index.php/IJET & Research Paper \\
\hline
\end{tabular}

\title{
A Study on improving the durability of drone according to the area of impact applied with finite element analysis method
}

\author{
Kye-Kwang Choi ${ }^{1}{ }^{*}$, Jae-Ung Cho ${ }^{2}$ \\ ${ }^{1}$ Department of Metal Mold Design Engineering, Kongju National University, 1223-24, \\ Cheonan Daero, Seobuk-gu, Cheonan-si, Chungnam, 31080, Republic of Korea \\ ${ }^{2}$ Division of Mechanical \& Automotive Engineering, Kongju National University, 1223-24, \\ Cheonan Daero, Seobuk-gu, Cheonan-si, Chungnam, 31080, Republic of Korea
}

\begin{abstract}
The objective of this paper is to identify the dangers of damage on drones according to the area of impact and obtain the basic data for improving the durability. The durability by impact according to the weight and speed of the large-size drone is calculated and analyzed using a finite element analysis method with 3D model according to the area of impact. For the analytical results, the possibility of fracture is identified and weak areas are improved through the distribution of equivalent stress and deformation analysis using polyester resin, which is a material used for the drone. The equivalent stresses applied to drones in head-on impact and broadside impact were 296.22MPa and 349.36MPa respectively. The broadside impact producing the highest fracture stress of over $300 \mathrm{MPa}$ and the bottom part of the battery pack is limited to a fairly narrow area, so the improvements can be made by reinforcing this area. The great damage may occur from rear impact as the results show $828.28 \mathrm{MPa}$, which is much higher than the fracture stress at rear impact to be the greatest drawback. Also for the deformation results, the values of head-on impact and broadside impact were in the safety range according to the elongation rate, while the drone greatly suffered from deformation and damage in rear impact. At the simulation analysis, the change of material must also be considered along with the change in design for rear collision. It is thought to obtain the basic data for future designing of large-sized drones by referring the results of this research, and it may contribute to the improvement of drone durability. By applying this study result to the drone, it is thought to contribute to the improvement on the durability of drone design due to the area of impact.
\end{abstract}

Keywords: Area of Impact; Drone; Durability; Equivalent Stress; Finite Element Method; Total Deformation

\section{Introduction}

The development of unmanned equipment has been continued over the past. Development has been progressed especially in narrow fields such as its use as military equipment. Typical examples include the UGV (unmanned ground vehicle), UAV (unmanned aerial vehicle), and USV(unmanned surface vehicle). UAV has especially been used recently by the public for aerial photography as a form of interest. Also, unlike the use of polyester resin in the past, they are made of various materials, which prove the successful case of popularization backed by the development of the material industry. They are called by drones and it is growing in scale from the growing participation of various industries (Durairaj M and Shathesh S., 2016), (J. U. Cho et al., 2009), (J. U. Cho et al., 2009), (Yang, C. S and Zhang, Y. J., 2012), (H. S. Cho and J. U. Cho., 2014), (Aminuddin W et al., 2016). However, drones used for free time cannot maintain a high level of altitude, which make them vulnerable to various obstacles, increasing the number of damages and accidents. Factors of collision include inexperienced operation and decrease of radio sensitivity in urban areas, and the motor and control module installed without an external case to increase the duration of flight. The frame of the drone or other important parts may be damaged even from small impact because of this structure (Pilipchuk V N et al., 2013), (Putti V S T et al., 2015), (H. P. Sun and J. U. Cho., 2015), (Alexis S J et al., 2015). We have set the Quadcopter type drone, which is the biggest and most general drone used for free time as the research model, and performed analysis using 3D Modeling. So, the research results of damage from obstacles head-on, broadside, and rear impact can be used as the basic data to improve the durability of the frame of drones.

\section{Research models}

The research model used in this research is shown in Fig. 1. The size of the research model is displayed as the diagonal length from the ends of each frame, and we have analyzed the $520 \mathrm{~mm}$ largesize Quadcopter as our subject. We have simplified both the upper part, which contains components for flight, and the lower section, which includes a camera and battery pack, into a box shape for analytical 3D modeling. We have reduced the time spent for analysis, and minimized error factors that may occur for detailed models. Fig. 2 displays the mesh configuration for applying the finite element analysis method, and the elements and number of joints turned out to be 33,917 and 21,759 respectively. Also, the property value of polyester resin that makes up drones is shown at Table 1. 

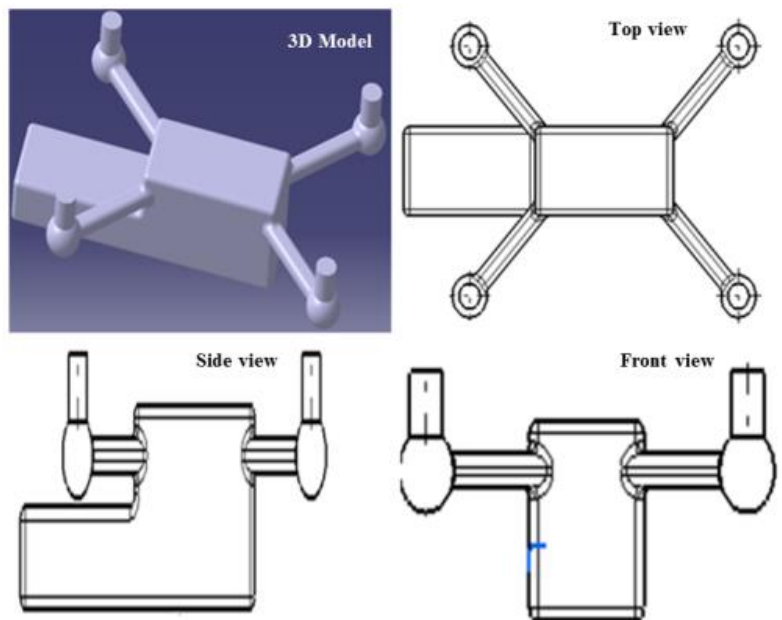

Fig. 1: Research Model.

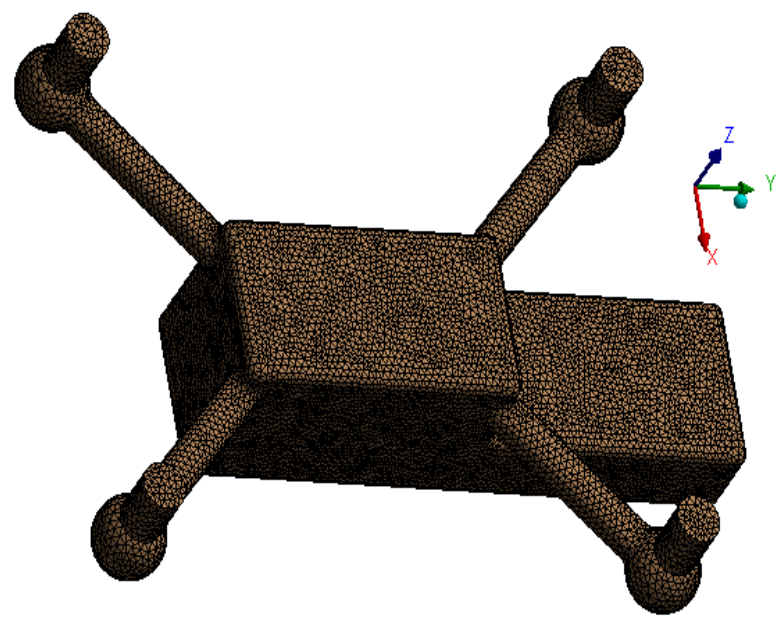

Fig. 2: Mesh of Research Model.

Table 1: Property of Material.

\begin{tabular}{ll}
\hline & Table 1: Property of Material. \\
\hline Material & Aluminum alloy \\
\hline Density $\left(\mathrm{kg} / \mathrm{m}^{3}\right)$ & 950 \\
Young's Modulus $(\mathrm{GPa})$ & 11 \\
Poisson's ratio & 0.33 \\
Yield strength $(\mathrm{MPa})$ & 280 \\
Ultimate strength $(\mathrm{MPa})$ & 300 \\
\hline
\end{tabular}

\section{Research method}

Fig. 3 shows the condition of analysis for head-on collision, Fig. 4 shows the condition of analysis for rear collision, and Fig. 5 shows the condition of analysis for broadside collision. The strength of applied condition of analysis is expressed in Eq. 1 and Eq. 2. The pressure that the drone receives during 0.01 seconds is calculated as $25000 \mathrm{~N}$ according to Eq. 1 and 2 assuming that the $15 \mathrm{Kg}$ drone collides with an object at a speed of $60 \mathrm{Km} / \mathrm{h} . \Delta \mathrm{P}$ is impulse $(\mathrm{kgm} / \mathrm{sec}), \mathrm{m}$ is mass $(\mathrm{kg}), v$ is velocity $(\mathrm{m} / \mathrm{s})$, and $\Delta \mathrm{t}$ is time $(\mathrm{sec})$.

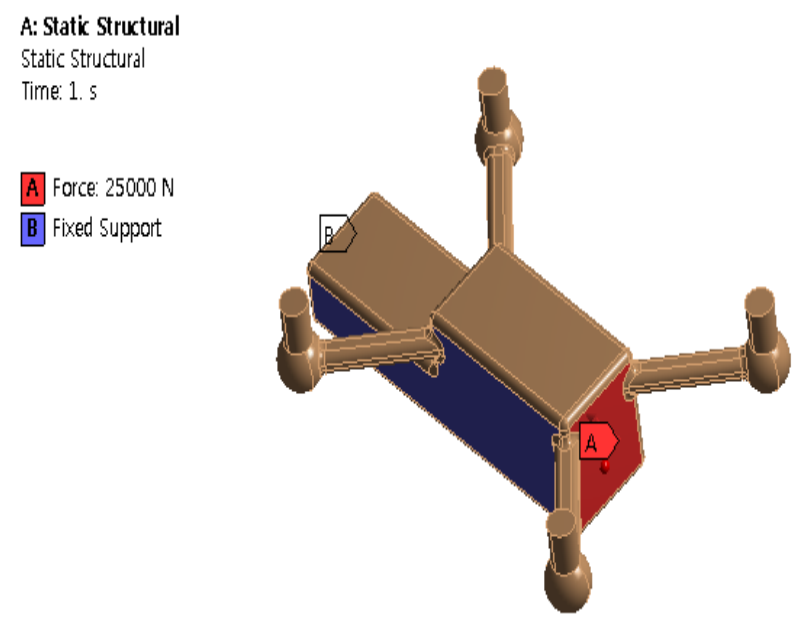

Fig. 3: Conditions for the Analysis of a Head-on Collision

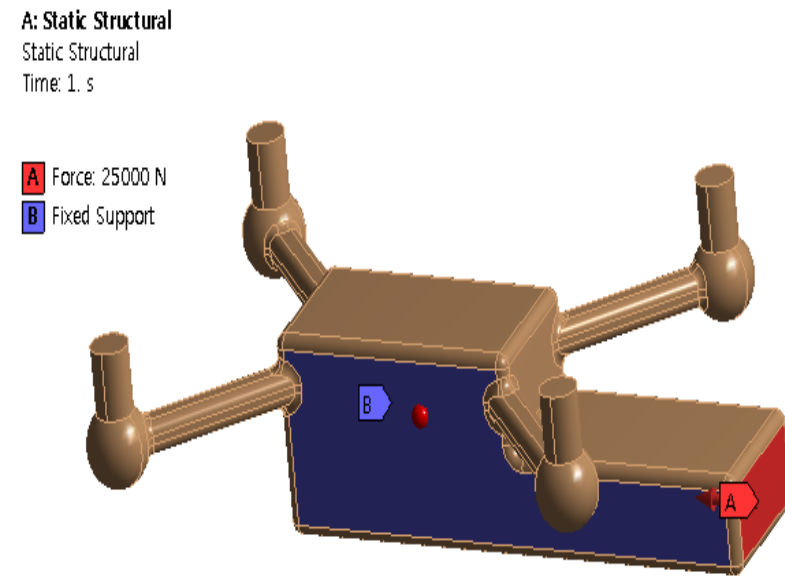

Fig. 4: Conditions for the Analysis of Rear Collision.

A: Static Structural

Static Structural

Time: 1.5

A Force: $25000 \mathrm{~N}$

B Fixed Support

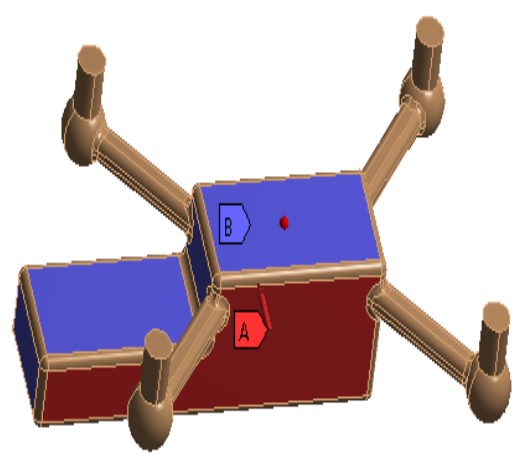

Fig. 5: Conditions for the Analysis of Broadside Collision.

$$
\begin{aligned}
\Delta P & =m \cdot v=(15) \times\left(60 \times \frac{1000}{3600}\right) \\
& =250 \mathrm{~kg} \cdot \mathrm{m} / \mathrm{sec}
\end{aligned}
$$

\section{Simulation analysis results}

\subsection{Analysis results of equivalent stress}

Fig. 6 shows the equivalent stress analysis results from head-on collision. Fig. 7 shows the results from rear collision of drones, and Fig. 8 shows the results from broadside collision. The maxi- 
mum level of equivalent stress from head-on collision in Fig. 6 turned out to be 296.22MPa. We can see that the upper part of the drone is dented from the effect of the head-on collision, and this means that the force is transferred to the inner part of the drone from the impact of head-on collision. The drone has not been displayed as damaged because of the assumption that the joints of each factor have not been separated, even though force higher than the material strength has been applied in the finite element analysis. However, we can assume that polyester, which is a brittle material, is damaged when looking at the internal shape of the model. We can approve that this model possesses a tolerant level of durability from the fact that the force from head-on collision did not reach the maximum level of $300 \mathrm{MPa}$.
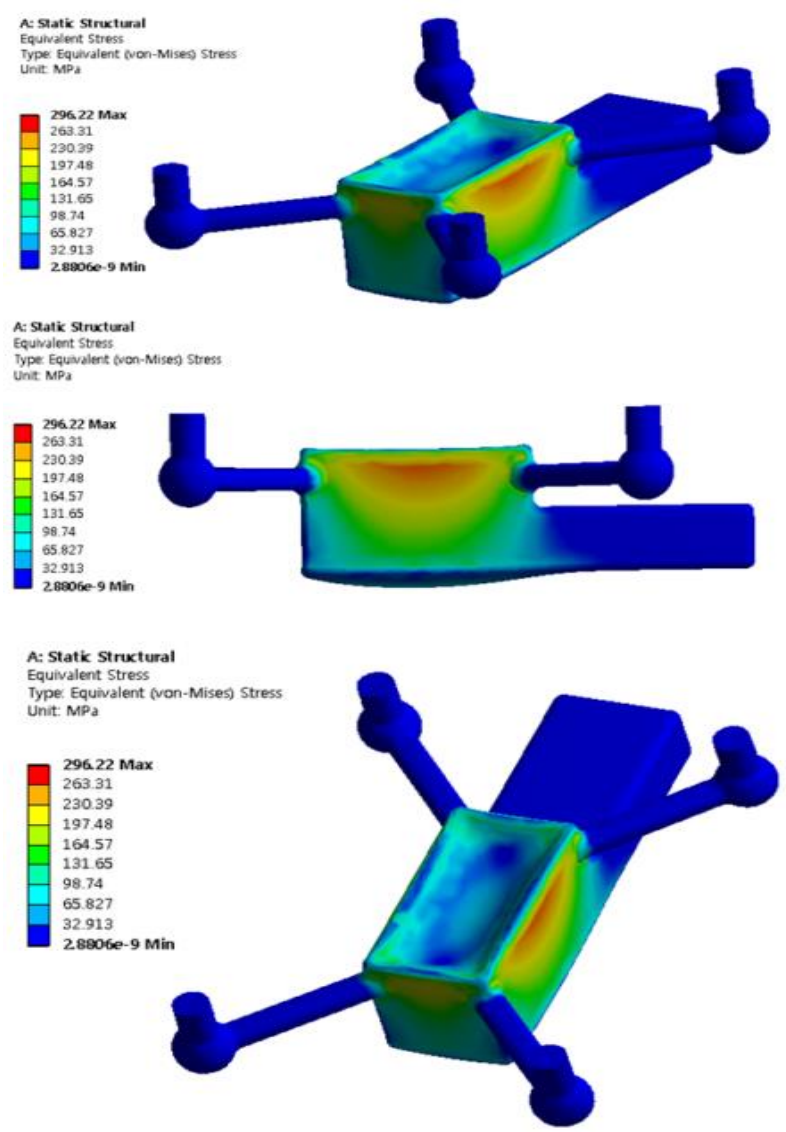

Fig. 6: Contour of Equivalent Stress on Research Model for Head-on Collisions.

Fig. 7 displays the equivalent stress from rear collision, and the maximum equivalent stress turned out to be $828.24 \mathrm{MPa}$. We can expect the damage to drones when facing rear collision of a stress of over a maximum level of $300 \mathrm{MPa}$, and predict damage to the entire frame through the transfer of stress. We can understand the need to improve the durability and change of material for rear collision of the analyzed model through reinforcement designs according to these analysis results.

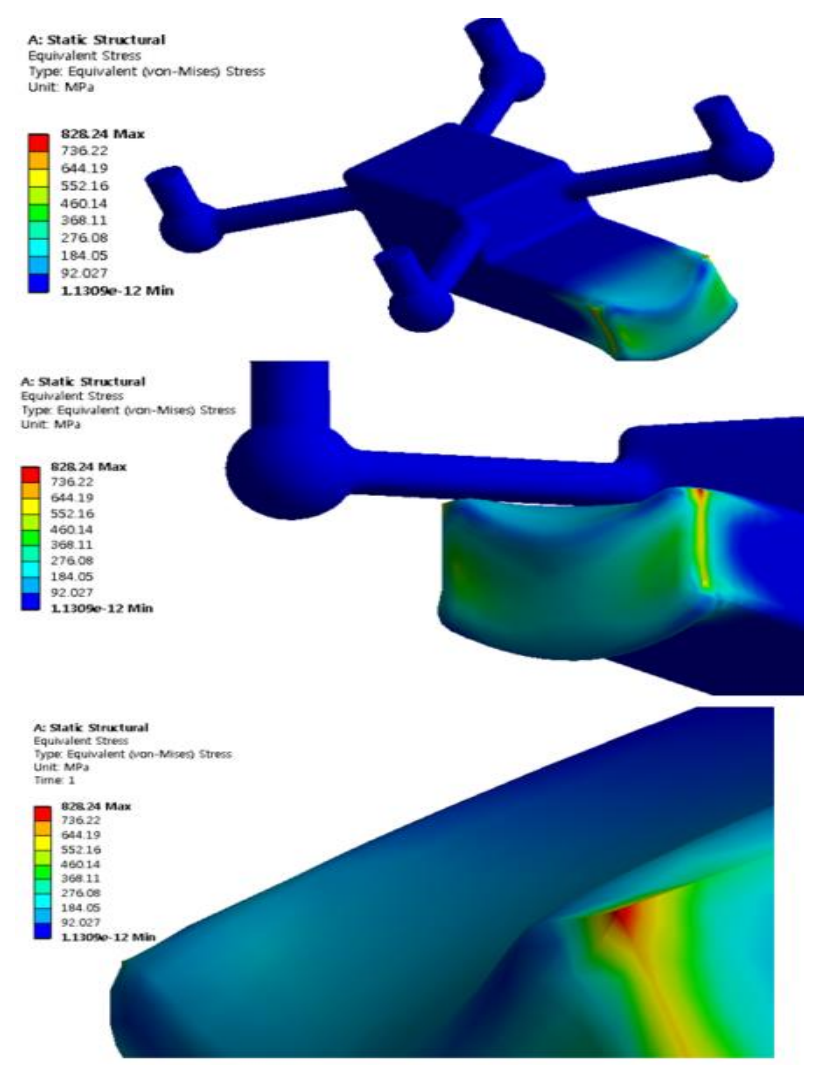

Fig. 7: Contour of Equivalent Stress on Research Model for Rear Collision.

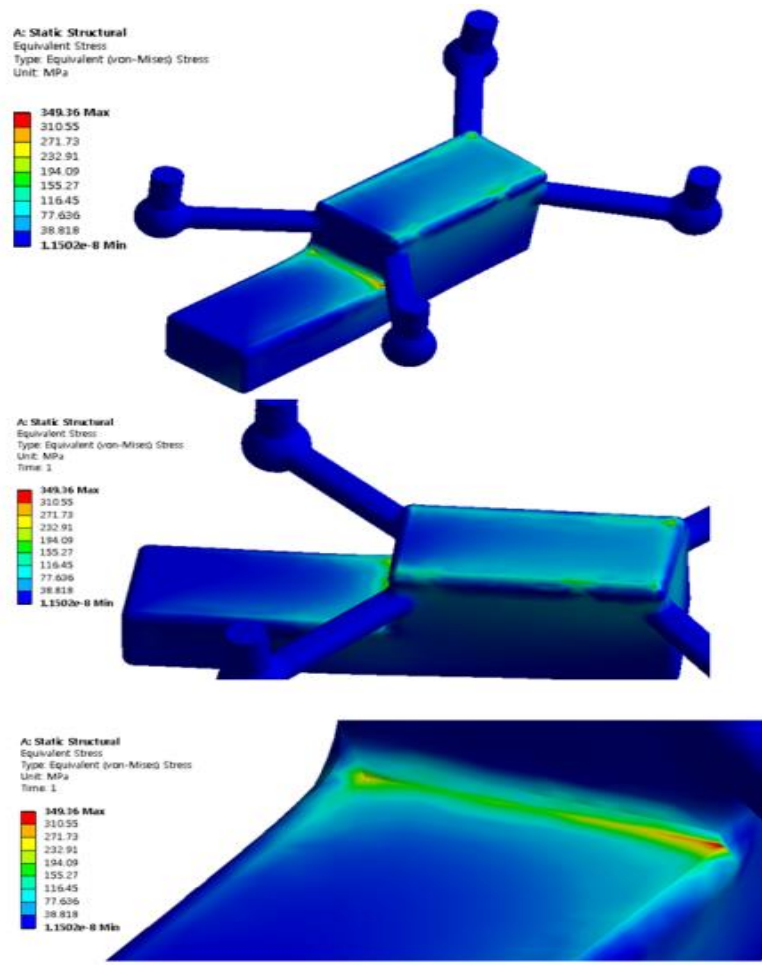

Fig. 8: Contour of Equivalent Stress on Research Model for Broadside Collision.

Fig. 8 displays the value of equivalent stress from broadside collision, and the maximum equivalent stress turned out to be 349.36MPa. Stress over the limit of material value has been observed, but we can see that stress is concentrated. We can predict that fracture will occur in the stress concentrated area, which is the connection between the flight component in the upper layer and the camera and battery pack of the lower layer. Thus, drones may obtain durability from broadside collision if reinforcement designs are made for this area. 


\subsection{Analysis results of deformation}

Fig. 9 displays deformation from head-on collision, Fig 10 shows deformation from rear collision, and Fig 11 shows deformation from broadside collision. First, the deformation from head-on collision in Fig. 9 turned out to be $4.0794 \mathrm{~mm}$, and the greatest level of deformation occurred in the front part of the motor support, which is responsible for the actual flight. However, it did not reach the level of fracture based on the elongation rate, so we can judge that it is within the range of repair on material.

Fig. 10 shows the deformation of rear collision, and a maximum deformation of $7.7944 \mathrm{~mm}$. Unlike the head-on collision, the deformation level turned out to be very high for both the upper and lower part from rear collision. We could see that the motor support of the upper part especially showed a high level of deformation, leading to a high risk of crash from the loss of flight ability from collision and damage. Thus, an improvement design is required in case of rear collision.

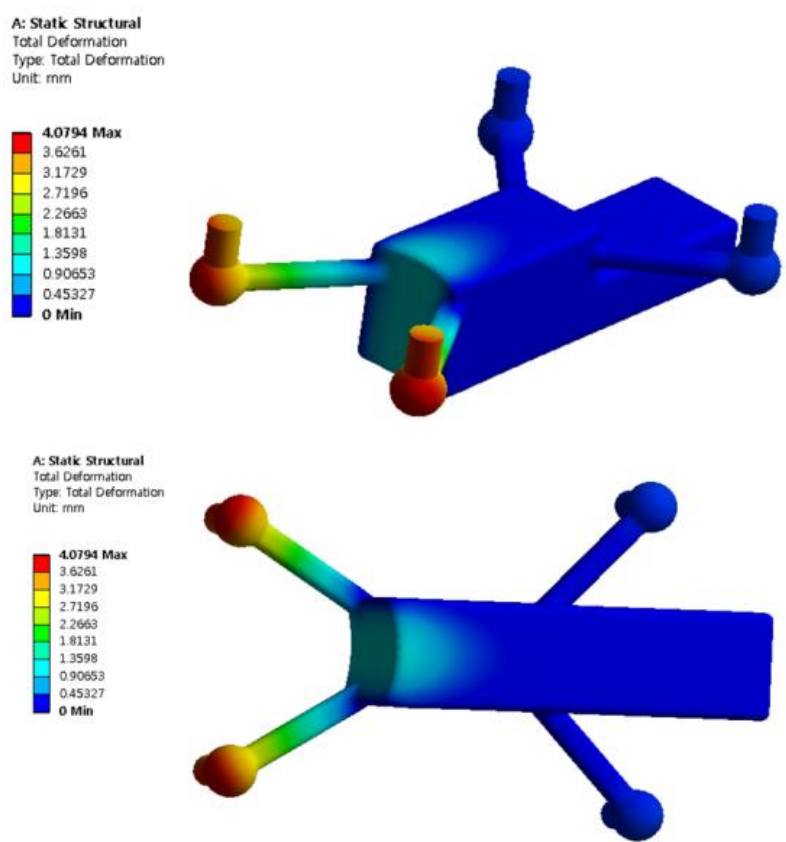

Fig. 9: Contour of Deformation on Research Model for Head-On Collisions.
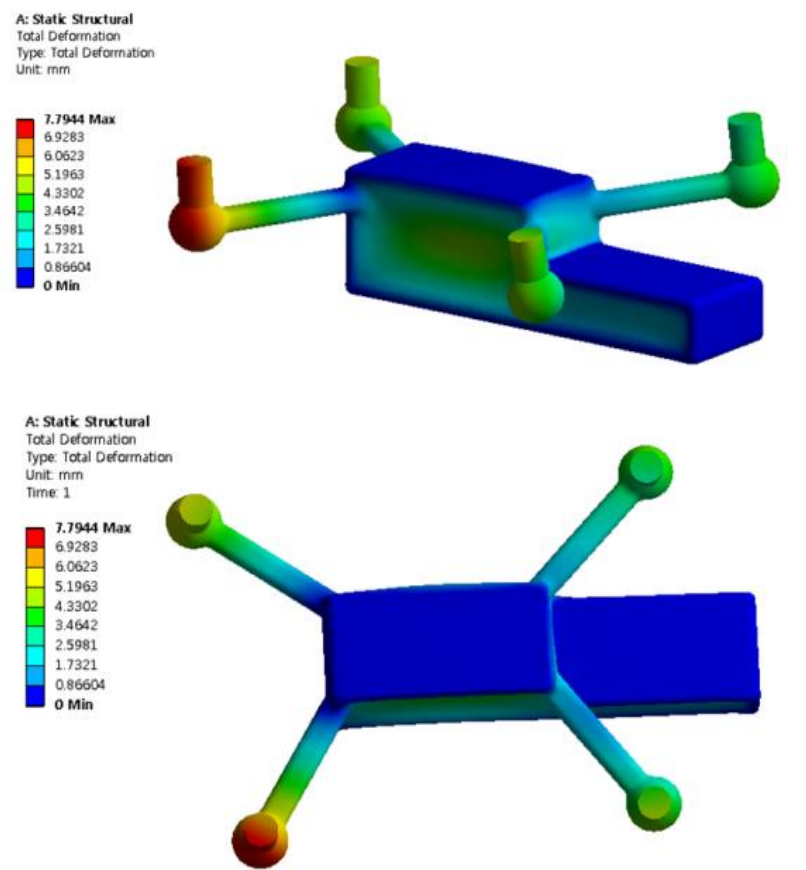

Fig. 10: Contour of Deformation on Research Model for Rear Collision.
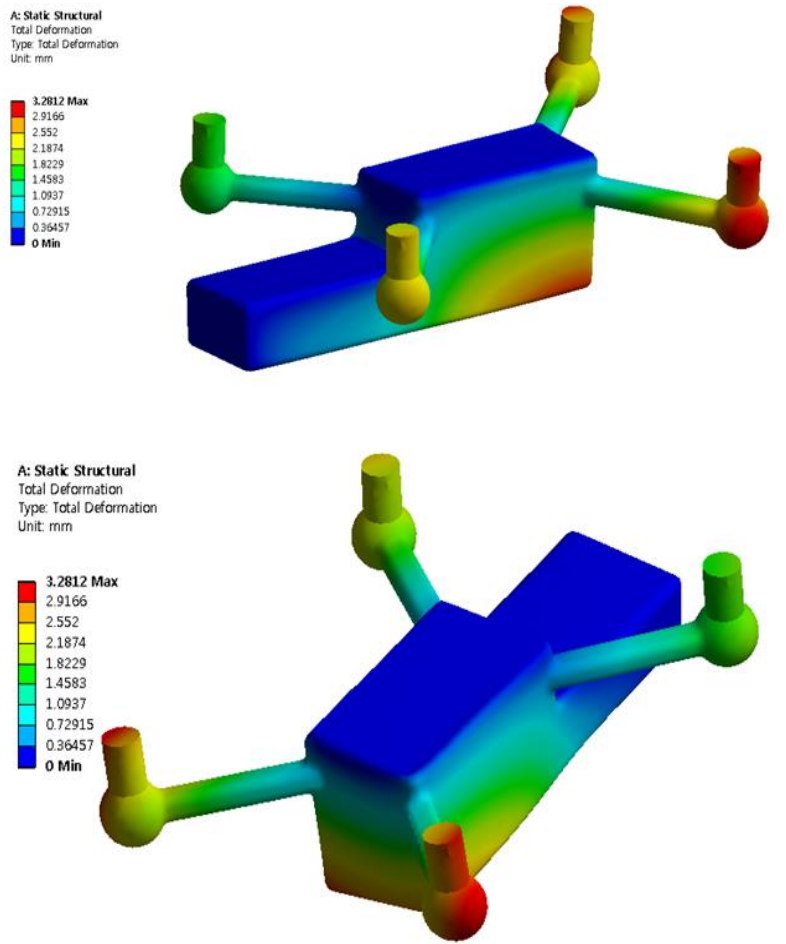

Fig. 11: Contour of Deformation on Research Model for Broadside Collision.

Fig. 11 shows the level of deformation from broadside collision, and the maximum deformation level turned out to be 3.2812. This is a relatively low result compared to the previous deformation results. However, the deformation seemed to be concentrated at the motor support and the lower part of the battery pack. Lithium polymer batteries are gradually being used for drones today, but lithium ion batteries are used more often because of its price. Lithium ion batteries are vulnerable to impact and have a risk of explosion, so changes such as attaching additional buffer material to the battery pack seem to be required.

\section{Conclusions}

In this study, the equivalent stress and deformation are analyzed with the finite element analysis method for the areas of impact of the $570 \mathrm{~mm}$ large-size drone composed of the assembly of an upper and lower layer. This result is concluded as follows;

1) The distribution of equivalent stress in head-on collision turned out to be $296.22 \mathrm{MPa}$, which is a value less than the fracture strength, making the durability of the research model reliable for head-on collision. However, the value is $828 \mathrm{MPa}$ for rear collision, which means that damage in the lower part of the drone including the camera, and a wide range of damage from stress transfer can be expected. Furthermore, the value of broadside collision is $349.36 \mathrm{MPa}$ and the stress is concentrated near the battery pack, which increases the risk of explosion. So, the improved design is required.

2) For the distribution of deformation according to each area of impact, the deformation value resulted in $4.0794 \mathrm{~mm}$ for head-on collision, which is an elongation rate within the safety range. Rear collision resulted in $7.7944 \mathrm{~mm}$, which led to damage in the motor support, loss in flight ability, and a high risk of crash. Also, similar to the results of the distribution of equivalent stress, the broadside collision led to a high risk of explosion from the damage of the battery pack, and a change in material strength or improvement design is required.

3) The drone possessed a sufficient level of durability for head-on collisions by the contour results of equivalent stress and deformation according to the area of impact, and ob- 
tained durability through improvement designs of battery packs for broadside collision. However, the change of material must be considered along with the change in design for rear collision.

4) We have been able to obtain base data for future designing of large-sized drones based on the results of this research paper, and it may contribute to the improvement of drone durability.

\section{References}

[1] Alexis S J, Gift M D M. and Kumar P S., 2015. Determination of Decisive Parameters in the Crack propagation Analysis of an Adhesive joint. Indian Journal of Science and Technology. 8 (35), pp.356-364

[2] Aminuddin W, Harunarashid H. and Ismail W, 2016. Estimating Emergency Department Maximum Capacity using Simulation and Data Envelopment Analysis. Indian Journal of Science and Technology. 9 (28): 1-10.

[3] C. D. Cho, M. S. Han and J. U. Cho., 2009. Study of dynamic crack propagation in 3PB steel specimens loaded by impact. International Journal of Precision Engineering and Manufacturing. 10(2): 67-72.

[4] Choi S, Han T, Ju W, Lee K and Wen J., 2012. Finite element analysis of a subsurface penny-shaped crack with crack-face contact and friction under a moving compressive load. Journal of Mechanical Science and Technology. 26 (9): 2719-26.

[5] Durairaj M, Shathesh S., 2016. An Intelligent Hybrid Mechanism to Predict the Risk of Cardio Vascular Disease. Indian Journal of Science and Technology. 9 (4): 1-8.

[6] Elijah G, Narasimha P B, Putti V S T and Prakash S, ,. 2015. Finite Element Analysis in Drilling GFRP Composite. Indian Journal of Science and Technology. 8 (15): 698-704

[7] Grace I, Ibrahim R. A. and Pilipchuk V. N., 2013. Low temperature brittle debond damage under normal compression of sandwich plates: Analytical modeling and experimental validation. Composite Structures. 98(1): 24-33.

[8] H. P. Sun, J. U. Cho., 2015. A Study on Static and Fatigue Fracture on Tapered Double Cantilever Beam Specimen with Aluminum Foam through Simulation and Experiment. Indian Journal of Science and Technology, 8 (26): 405-410

[9] H. S. Cho and J. U. Cho., 2014. The characteristics of shear adhesive interface fracture of aluminum foam DCB bonded using a single-lap method. International Journal of Precision Engineering and Manufacturing. 15(7): 1345-1350.

[10] Yang, C. S and Zhang and., 2012. FEM analyses for influences of stress-chemical solution on THM coupling in dual-porosity rock mass. Journal of Central South University. 19(4): 1138-1147. 